\title{
Single particle tracking of dynamically localizing TatA complexes in Streptomyces coelicolor
}

\author{
Katherine Celler, Gilles P. van Wezel* , Joost Willemse \\ Molecular Biotechnology, Institute of Biology Leiden, Sylvius Laboratories, Leiden University, Sylviusweg 72, 2333 BE Leiden, The Netherlands
}

\section{A R T I C L E I N F O}

\section{Article history:}

Received 28 June 2013

Available online 15 July 2013

\section{Keywords:}

Live imaging

Dynamic protein localization

Particle tracking

Cell biology

Twin arginine translocation (Tat)

\begin{abstract}
A B S T R A C T
The Tat (twin-arginine translocation) pathway transports folded proteins across the bacterial cytoplasmic membrane and is a major route of protein export in the mycelial soil-dwelling bacterium Streptomyces. We recently examined the localization of Tat components (TatABC) in time-lapse imaging and demonstrated that all three components colocalize dynamically with a preference for apical sites. Here we apply an in-house single particle tracking package to quantitatively analyze the movement of the TatA subunit, the most abundant of the Tat components. Segmentation and analysis of trajectories revealed that TatA transitions from free to confined movement and then to fixed localization. The sequence starts with a mixed punctate and dispersed localization of TatA oligomers, which then develop into a few larger still foci, and finally colocalize with TatBC to form a functional translocation system. It takes $15-30$ min for the Tat export complex to assemble and most likely become active. With this study we provide the first example of quantitative analysis of dynamic protein localization in Streptomyces, which is applicable to the study of many other dynamically localizing proteins identified in these complex bacteria.
\end{abstract}

(c) 2013 Elsevier Inc. All rights reserved.

\section{Introduction}

In recent years, high resolution cell-biology techniques have revealed that, despite their small size, bacteria have a complex internal organization [1-3]. Molecular crowding within cells results in a dynamic equilibrium of components [4], leading to their non-uniform spatial distribution [5]. This necessitates diffusion or active transport to areas where proteins can function, such as in nuclear organization, cell division or differentiation [6,7]. In vivo multidimensional fluorescence microscopy allows scientists to visualize these processes, which often display rapid anddirected spatial relocation of proteins in three-dimensional time lapse movies [8-10]. Taking cell division proteins as an example, these have been seen oscillating from one end of the cell to the other, forming rings across its midsection, or focal complexes at specific intracellular sites [11-14]. To better understand these modes of action, and the lifespan of cellular proteins, quantitative measurements of protein abundance and dynamics are necessary.

A challenging subject for the study of dynamic protein localization is the Gram-positive filamentous soil bacterium Streptomyces. These mycelial organisms are used as natural producers of a large number of commercially important secondary metabolites, enzymes, and other secreted protein products $[15,16,20]$. In most bacteria the Sec pathway is the predominant route for protein

\footnotetext{
* Corresponding author.

E-mail address: g.wezel@biology.leidenuniv.nl (G.P. van Wezel).
}

export, but the streptomycetes encode an unusually large numbers of Tat (twin-arginine translocation) substrates [26-28]. During fermentation, growth of hyphae is a balance of tip extension, branching frequency and fragmentation [17,18], all of which affect the efficiency of production and secretion. Fragmentation of hyphae strongly enhances protein secretion [24], which correlates well with our observation that the components of the Tat pathway in Streptomyces localize at the apical sites of hyphae [25]. A better understanding of where and when secretion takes place in the hyphae can lead to important insights for strain-improvement and rational process design.

The Streptomyces Tat machinery consists of Tat $\mathrm{A}$, TatB and TatC proteins $[28,29]$, functional homologs of proteins found in Gramnegative bacteria such as Escherichia coli [30]. The tetrameric TatA is the most abundant component of the Tat complex [31] and many copies of this monotopic membrane protein are believed to cluster around a substrate-bound TatBC complex to bring about the transport of a folded protein across the membrane, with an average of $\sim 25$ TatA subunits per complex [32-34].

Analysis of the Tat complex using live imaging revealed that the proteins localize surprisingly dynamically throughout the life cycle [25]. The dynamic localization of the separate components, however, creates difficulty in understanding their biological implication. Quantitative description and modeling of these movements by single particle tracking is therefore necessary to better understand complex assembly in time and space. Here we provide a detailed analysis of the dynamics of the TatA component of the 
Tat protein export pathway, using an in-house foci tracking package which was developed in the frame of the Particle Tracking Challenge of the International Symposium on Biomedical Imaging (ISBI) in 2012 (Chenouard et al., submitted for publication). Specific questions we wished to address were (1) whether TatA undergoes different stages of motion prior to co-localization with TatB and TatC; (2) if so, what are the durations of these transitional stages; and (3) what is the time it takes for the complex toassemble.

This is the first mathematical tracking analysis of diffusion-limited foci in vegetative and aerial hyphae of Streptomyces, which can be applied to study the movement of many other dynamic proteins in these complex microorganisms.

\section{Material and methods}

\subsection{Strains and culturing conditions}

Strain BRO3 is a derivative of S. coelicolor FM145, a variant of $S$. coelicolor M145 with reduced autofluorescence [35]. BRO3 produces TatA-eGFP from the native chromosomal location (FM145, TatA::TatA-eGFP). Construction of the strain is described elsewhere [25]. Initial examination of all strains was done by stereo fluorescence and light microscopy with a Zeiss Lumar V12 as described previously [38]. To culture samples for live imaging, uncoated $\mu$ dishes (Ibidi $\mathrm{GmbH}$ ) were perforated at the side while closed tightly, and subsequently were semi-filled with SFM medium [36]. These dishes were inoculated with $1 \mu \mathrm{L}$ of spores at a concentration of $10^{9}$ spores $/ \mathrm{mL}$, turning the lid so that it was supported on the vents, allowing for gas exchange, and were sealed off by two layers of Parafilm to prevent drying of the medium. Samples were incubated at $30^{\circ} \mathrm{C}$ for $48 \mathrm{~h}$ before being transferred to the live imaging microscope. Temperature was controlled with a p-insert heating block and kept at $30^{\circ} \mathrm{C}$, and allowed to calibratefor $1 \mathrm{~h}$ before commencing imaging.

\subsection{Data collection}

\subsubsection{Fluorescence microscopy}

Fluorescence and corresponding light micrographs were obtained with a Zeiss Observer inverted fluorescence microscope (with an Hamamatsu CCD camera at a resolution of $78 \mathrm{~nm} /$ pixel) as described $[35,37]$. The green fluorescent images were created using 470/40-nm bandpass excitation and 525/50 bandpass detection. All images were background-corrected, setting the signal outside the hyphae to 0 to obtain a sufficiently dark background. These corrections were made using Axiovision software 4.8.

\subsubsection{Time-lapsed (live) imaging}

Imaging was performed with a Zeiss Observer A1 microscope with a Hamamatsu EM-CCD C9100-02 camera as described [39]. Images were taken at 2 min intervals with an exposure time of $100 \mathrm{~ms}$. In order to minimize focal drift, the microscope stage and imaging chamber were allowed to equilibrate for $60 \mathrm{~min}$ before imaging. Initially z-stacks of five images with a focal depth of $0.5 \mu \mathrm{m}$ were taken to prevent out-of-focus movement of the hyphae. These image sequences were then z-projected using the average z-stack projection method implemented in ImageJ.

\subsubsection{Computer programs}

The particle tracking algorithm was submitted to the Particle Tracking Challenge (http://bioimageanalysis.org/track/) of the 2012 International Symposium on Biomedical Imaging (http:// www.biomedicalimaging.org/2012/), in which the performance of existing and newly developed particle tracking algorithms was tested against synthetic image sets with ground truth data. The methods and results of the challenge have been described (Chenouard et al., submitted for publication). For details on the algo- rithm see the Supplementary Methods. The algorithm was implemented in the Java programming language (Sun Microsystems Inc., Santa Clara, CA) in the form of a plug-in for Image (National Institutes of Health, Bethesda, MD), the computer-platform independent public domain image analysis program inspired by NIHImage. ImageJ version 1.460 was used with the Java 1.7 compiler.

\section{Results and discussion}

We focused on TatA complex assembly as a whole, which occurs at a timescale several orders of magnitude greater than the fast movement of individual proteins. To capture an entire localization sequence (with an initial estimated duration of roughly 30$60 \mathrm{~min}$ ) prior to bleaching of the samples, we took images for up to $3.5 \mathrm{~h}$ at $2 \mathrm{~min}$ intervals. Since mobility data suggests that cytosolic diffusion occurs at a time scale two orders of magnitude faster than membrane diffusion, we observed the dynamics of slow-moving membrane-bound macrocomplexes, and not the cytosolic fraction of TatA. S. coelicolor FM145, a derivative from the wild-type strain withreduced autofluorescence [35], was used for the localization studies, allowing imaging of the fluorescently labeled proteins at significantly higher signal to noise ratio. In 18 time lapse imaging movies of diffusion-limited TatA-eGFP, roughly 1500 foci were tracked from the onset of imaging to final localization. Foci tracked/detected in only two consecutive frames were not taken into account. The distribution of tracks (Fig. 1) demonstrates that short tracks predominate, with roughly half of all tracks corresponding to 10 or less consecutive frames.

Our previous study revealed increased amounts of localization in aerial hyphae, and imaging was therefore performed on early aerial hyphae after $48 \mathrm{~h}$ of growth. At this point, many hyphae had already stopped growing due to crowding in the sample, butoccasionally extending tips were still observed. In the growing hyphae, the Tat complex was seen following the tips.

The broad range of stoichiometries exhibited by TatA suggests that dynamic polymerization and depolymerization occurs during assembly [33]. This is evident in our time lapse sequences, where protein complexes display different types or stages of movement prior to fixed localization (ex. Fig. 2 and Supplementary Video 1). At the start of imaging, mainly dispersed fluorescence is seen, with gradually many faint foci appearing to 'race' along the hyphae. During this fast movement, several strong fluorescent foci become visible localizing (mainly) near the tips at roughly a $2 \mu \mathrm{m}$ distance. Over the course of the time lapse, the faint focieither fade or merge with larger stable fluorescent foci. These faint foci have short tracks lasting only 3-4 frames or 6-8 min. Particle tracking highlights these tracks as brief directed bursts of motion. The larger foci remain stationary, or wobble before stabilizing.

A large variation in fluorescence intensity is seen over the course of imaging. Initial faint foci assemble over the course of the time lapse imaging to form complexes with increased fluorescent intensity, likely reflecting natural protein oligomerization. Although formation of protein complexes can be enhanced by an eGFP fusion, which may affect protein functionality [40], we previously demonstrated that the TatA fusion is fully functional, restoring secretion of Tat substrates to wild-type levels. For full functional analysis of the TatA fusion product and Tat complex formation we refer to our previous work [25].

Supplementary Video 2 shows another representative example of dynamic localization. The final image from the time lapse is presented (Fig. 3A), with the detected TatA-eGFP tracks projected onto the image. Despite the fading of foci due to the imaging, three tracks could be followed for the entire duration $(1 \mathrm{~h})$ of the movie. The trajectories of these tracks were plotted (Fig. 3B), demonstrating representative trajectories of a still focus, a rapidly traveling focus (which becomes confined after $10 \mathrm{~min}$ ) and a wobbling focus. 


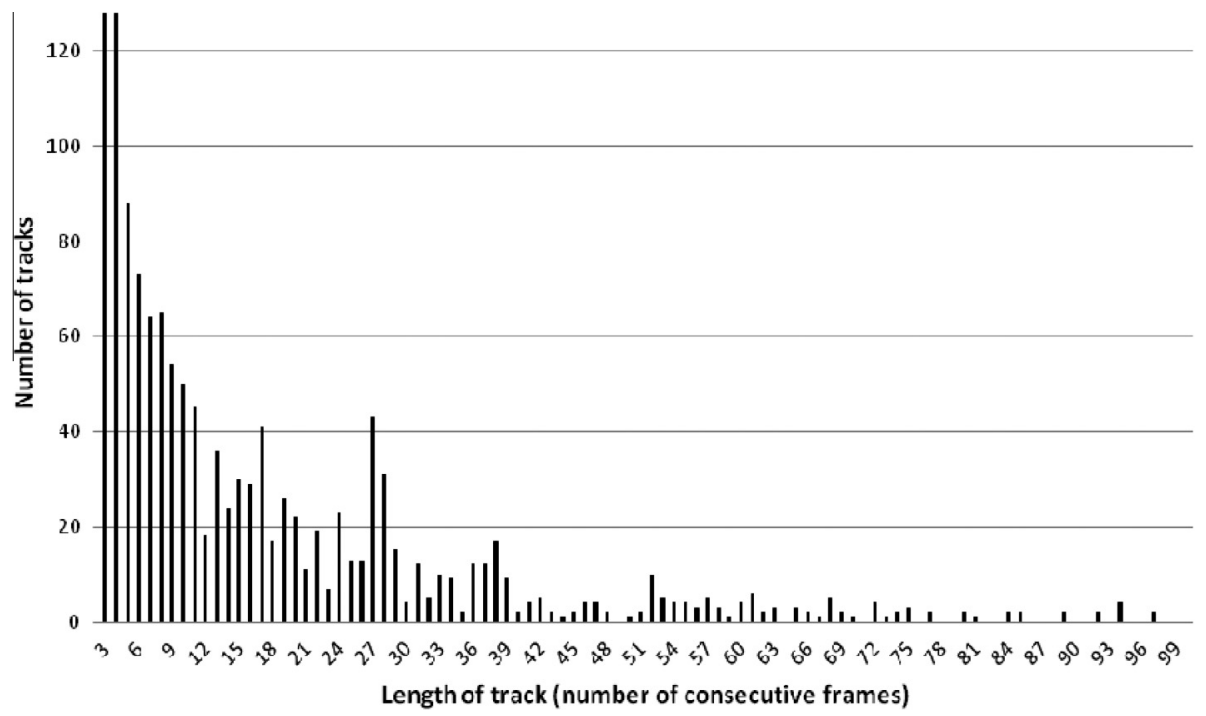

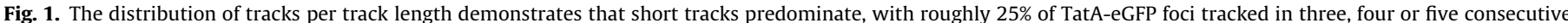

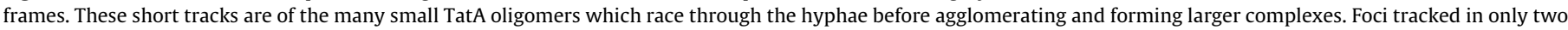
frames were not taken into account by the software. Roughly $50 \%$ of tracks are 10 consecutive frames or shorter.
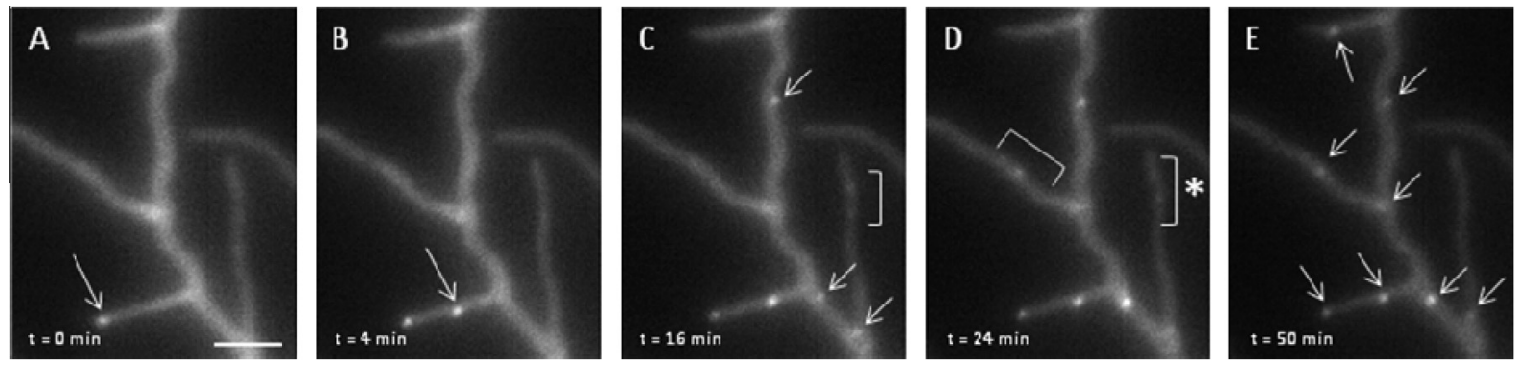

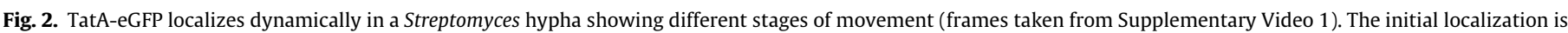

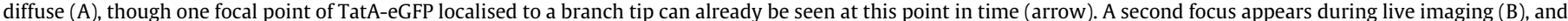

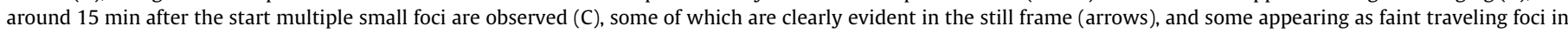

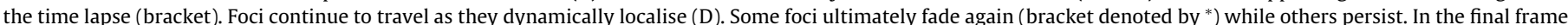

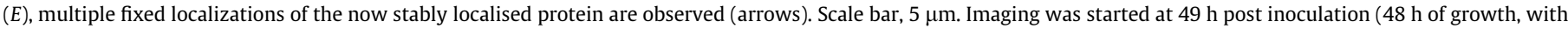
$1 \mathrm{~h}$ of incubation time in the microscope). This time was deemed $t=0$.

Plotting of the mean square displacement (MSD, see Supplementary data S1) versus time for thesetracks (Fig. 3C) facilitated classification of the different modes of motion observed. In this case, MSD curves reflect the global changes that occur during complex assembly, and not rapid-scale protein dynamics. In the case of the wobbling focus, the MSD is irregular and does not show a clear trend towards a particular mode of diffusion. The trajectory of the traveling focus appears to consist of two different stages, namely an initial phase of rapid diffusion followed by a phase of slower diffusion. The still focus has an MSD curve with a slope of zero. Analysis was done for the first half of time lags for these curves, or 30 min, to minimize error.

Supplementary Video 3 shows a third example of the dynamic localization patterns described above (for still images see Fig.4). The tracks of detected foci projected onto the image (Fig. 4A). In this movie, focus 1 (circled in Fig. 4B) hardly moves over the length of the time-lapse; the MSD curve has a slope of zero. Focus two initially moves at greater speed (in the direction of the arrow, Fig. 4B) than the other foci in a seemingly directed manner. It then stalls before continuing motion and later settling in a fixed position; from its MSD curve (Fig. 4C) the focus is seen to undergo different motion regimes. The multiple foci labeled in a region three seem to wobble and by eye fall into the same category of motion. When their MSD is plotted, however, it is clear that they have different rates of diffusion.

We obtaineda sufficiently large number of 'full tracks' to obtain reliable insight into how TatA complexes localize, starting with diffuse localization, followed by assembly and finally settlement. Track lengths for TatA-eGFP in the movies varied from foci linked in a few consecutive frames to tracks that could be followed for several hours (>90 linked foci). Our observations from the live imaging experiments suggest that the categories of motion exhibited by TatA (still, traveling or wobbling) are stages in a single localization sequence. Proteins may be observed in each of the different stages of motion in a single time lapse since dynamics within the hyphae are not synchronized. Short tracks represent foci that fade or fuse at the onset of imaging, while longer tracks undergo dynamic transition through all stages of movement. The latter start with dispersed localization, with faint foci likely consisting of only a few TatA tetramers, followed by confined movement when tetramers oligomerize, and finally a fixed localization, at which point TatA multimers most likely colocalize with TatBC to form a functional translocation sys- 

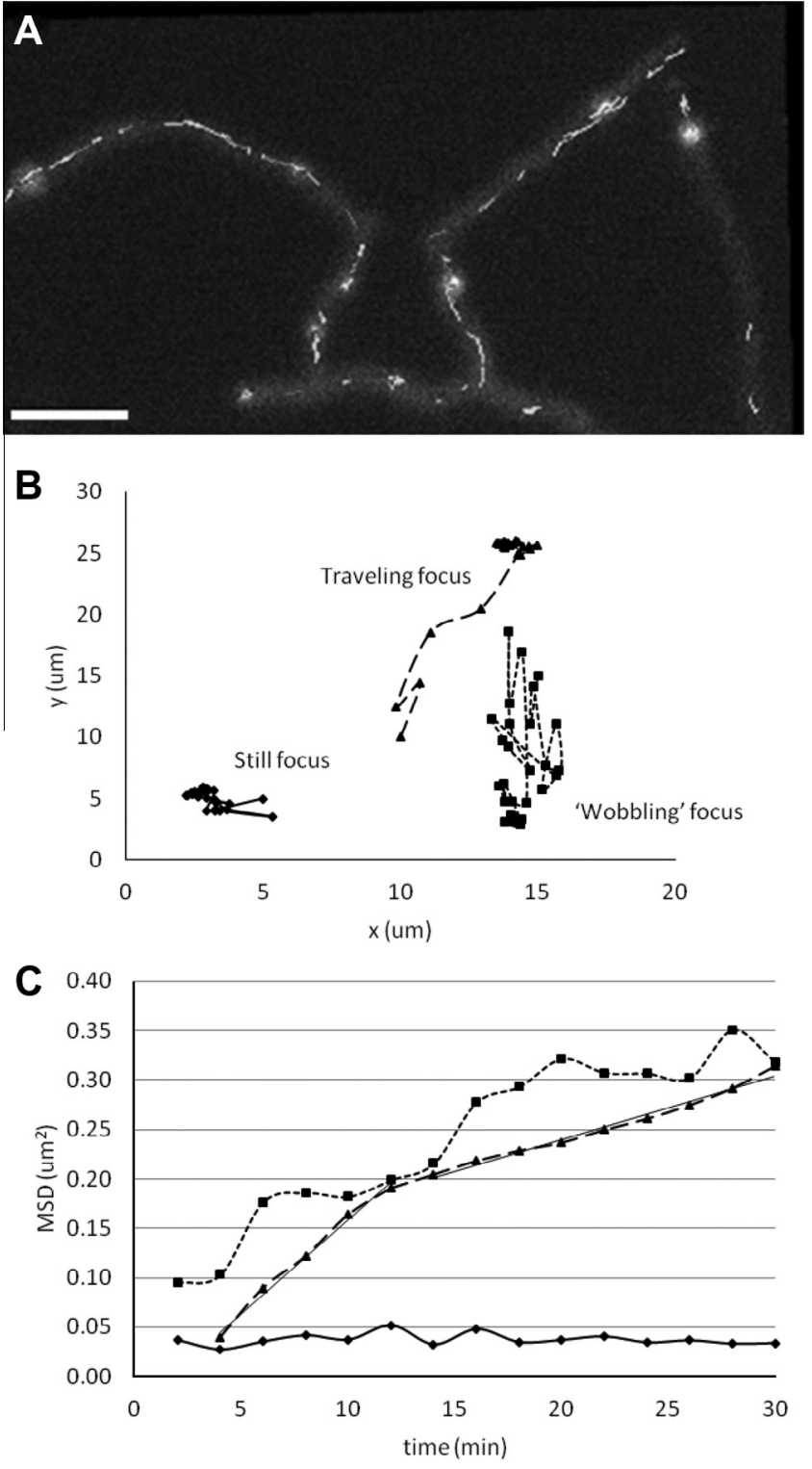

Fig. 3. A trajectory and MSD analysis of dynamic TatA-eGFP localization in a Streptomyces hypha provides evidence for different stages of localization (frame taken from Supplementary Video 2). TatA-eGFP tracks projected onto the frame demonstrate that dynamics occur throughout entire hyphae (A). Scale bar, $5 \mu \mathrm{m}$. Foci could be classified into those that are still, traveling and wobbling (B). the displacement of these foci is plotted on an $x-y$ plot, with tracks starting at $(5,5)$ $(10,10)$ and $(15,15)$, respectively. The still focus stays close to its origin; the traveling focus appears to move rapidly in a directed fashion for the first part of its trajectory and then to localise; the wobbling focus has a more random trajectory. The MSD is plotted versus time for the foci shown (C). Only the first half of the curve (total time $60 \mathrm{~min}$ ) is plotted and taken into consideration to minimise error. The still focus has a slope of zero. The traveling focus has an MSD curve with two regimes: fast, followed by slower diffusion. Linear trend lines are fitted to these two regimes. The MSD of the wobbling focus has an upward trend, but no conclusions can be drawn on the diffusion.

tem. By taking into account only the foci that undergo all of the above stages of localization, the average time for a focus of TatA to assemble and find its proper position was estimated at between 15 and 30 min (Supplementary Fig. 1).

This localization time may be indicative of the time required for an active Tat $A B C$ transport complex to assemble. The current model for the Tat mechanism postulates that TatA polymerizes in response to substrate binding to the TatBC complex. The decrease in mobility during localization supports this idea and most likely represents the formation of a TatABC complex which actively
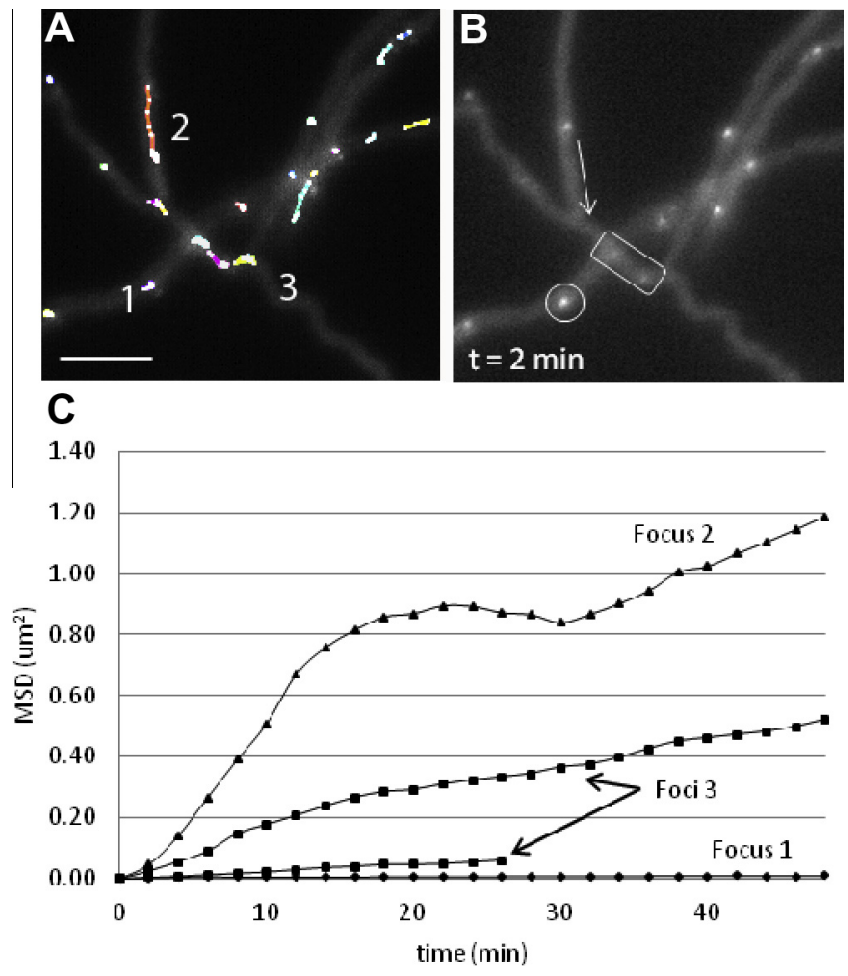

Fig. 4. TatA-eGFP localizes dynamically in a Streptomyces hypha showing different stages of movement (frames taken from Supplementary Video 3). Detected tracks of the foci are projected onto the frame with different colours (A). Some foci are still (1), some travel linearly before stalling, continuing motion, and then become still (2), and some wobble backwards and forwards (3), For clarity, the still focus is circled, the direction of travel of focus 2 is shown with an arrow, and the portion of a hyphae where several foci (foci ' 3 ') are wobbling is denoted by a rectangle (B). Scale bar, $5 \mu \mathrm{m}$. The MSD is plotted versus time for the foci presented (C). Only the first half of each curve is plotted and taken into consideration to minimise error. The MSD of the still focus is zero. The traveling focus, which travels quickly, stalls, and then continues motion until localizing has an MSD with different regimes. Two foci from region three show different rates of diffusion.

transports proteins outside the bacterial cell [33]. Our time lapse imaging in Streptomyces supports the notion that Tat component dynamics are very complex, with the proteins undergoing various phases of motion (and consequently rates and modes of diffusion) during localization.

Previous studies on localization of the replisome machinery in Streptomyces observed that most apically localized DNA replisomes were more than $1.5 \mu \mathrm{m}$ from the tip [41], settling at a position on average $5.32( \pm 2.00) \mu \mathrm{m}$ from the tip [42]. Our unpublished data by high resolution electron microscopy further suggest that the very tips of hyphae are a ribosome-free area, which could also explain why the Tat complex localizes near, but not at the tip. Ribosomes are known to localize near the nucleoids, and these data would confirm a model coupling transcription with translation and subsequent secretion of certain protein products by the Tat system.

To the best of our knowledge, our analysis of TatA dynamics is the first example of a quantitative analysis of dynamic protein localization in Streptomyces. Study of other dynamically localizing fluorescent proteins will provide more insight into the biochemical details of protein assembly and localization. Tracking single proteins in the cell and analyzing their trajectories will significantly increase our understanding of dynamic behavior of proteins in vivo.

\section{Acknowledgments}

We are grateful to the organizers of the ISBI Particle Tracking Challenge 2012 for stimulating discussions. We would like to thank 
Tracy Palmer for providing us with the TatA-eGFP fusion constructs and for her helpful comments on the manuscript. This work was supported by a VICI grant from the Netherlands Applied Research Council (STW) to GPvW.

\section{Appendix A. Supplementary data}

Supplementary data associated with this article can be found, in the online version, at http://dx.doi.org/10.1016/j.bbrc.2013.07.016.

\section{References}

[1] K. Celler, R.I. Koning, A.J. Koster, G.P. van Wezel, Multidimensional view of the bacterial cytoskeleton, J. Bacteriol. 195 (2013) 1627-1636.

[2] D. Lopez, R. Kolter, Functional microdomains in bacterial membranes, Genes. Dev. 24 (2010) 1893-1902.

[3] W. Margolin, Sculpting the bacterial cell, Curr. biol.: CB 19 (2009) R812-822.

[4] R.J. Ellis, Macromolecular crowding: obvious but underappreciated, Trends Biochem. Sci. 26 (2001) 597-604.

[5] J. Capoulade, M. Wachsmuth, L. Hufnagel, M. Knop, Quantitative fluorescence imaging of protein diffusion and interaction in living cells, Nat. Biotechnol. 29 (2011) 835-839.

[6] J.R. McCormick, K. Flardh, Signals and regulators that govern Streptomyces development, FEMS Microbiol. Rev. 36 (2012) 206-231.

[7] R.M. Figge, A.V. Divakaruni, J.W. Gober, MreB, the cell shape-determining bacterial actin homologue, co-ordinates cell wall morphogenesis in Caulobacter crescentus, Mol. Microbiol. 51 (2004) 1321-1332.

[8] J.T. Mika, B. Poolman, Macromolecule diffusion and confinement in prokaryotic cells, Curr. Opin. Biotechnol. 22 (2011) 117-126.

[9] P. Meyer, J. Dworkin, Applications of fluorescence microscopy to single bacterial cells, Res. Microbiol. 158 (2007) 187-194.

[10] L. Shapiro, H.H. McAdams, R. Losick, Why and how bacteria localize proteins, Science 326 (2009) 1225-1228.

[11] H.P. Erickson, D.E. Anderson, M. Osawa, Ftsz in bacterial cytokinesis: cytoskeleton and force generator all in one, Microbiol. Mol. Biol. Rev. 74 (2010) 504-528.

[12] Z. Hu, J. Lutkenhaus, Topological regulation of cell division in Escherichia coli involves rapid pole to pole oscillation of the division inhibitor MinC under the control of MinD and MinE, Mol. Microbiol. 34 (1999) 82-90.

[13] E.E. Noens, V. Mersinias, J. Willemse, B.A. Traag, E. Laing, K.F. Chater, C.P. Smith, H.K. Koerten, G.P. van Wezel, Loss of the controlled localization of growth stage-specific cell-wall synthesis pleiotropically affects developmental gene expression in an ssgA mutant of Streptomyces coelicolor, Mol. Microbiol. 64 (2007) 1244-1259.

[14] D.M. Raskin, P.A. de Boer, Rapid pole-to-pole oscillation of a protein required for directing division to the middle of Escherichia coli, Proc. Natl. Acad. Sci. USA 96 (1999) 4971-4976.

[15] D.A. Hopwood, Streptomyces in nature and medicine: the antibiotic makers, Oxford University Press, New York, 2007.

[16] S. Horinouchi, Mining and polishing of the treasure trove in the bacterial genus streptomyces, Biosci. Biotechnol. Biochem. 71 (2007) 283-299.

[17] K. Celler, C. Picioreanu, M.C. van Loosdrecht, G.P. van Wezel, Structured morphological modeling as a framework for rational strain design of Streptomyces species, Antonie Van Leeuwenhoek 102 (2012) 409-423.

[18] L. Nieminen, S. Webb, M.C. Smith, P.A. Hoskisson, A flexible mathematical model platform for studying branching networks: experimentally validated using the model actinomycete, Streptomyces coelicolor, PLoS One 8 (2013) e54316.

[20] G.P. van Wezel, K.J. McDowall, The regulation of the secondary metabolism of Streptomyces: new links and experimental advances, Nat. Prod. Rep. 28 (2011) 1311-1333.

[24] G.P. van Wezel, P. Krabben, B.A. Traag, B.J. Keijser, R. Kerste, E. Vijgenboom, J.J. Heijnen, B. Kraal, Unlocking Streptomyces spp. for use as sustainable industrial production platforms by morphological engineering, Appl. Environ. Microbiol. 72 (2006) 5283-5288.

[25] J. Willemse, B. Ruban-Osmialowska, D. Widdick, K. Celler, M.I. Hutchings, G.P. van Wezel, T. Palmer, Dynamic localization of Tat protein transport machinery components in Streptomyces coelicolor, J. Bacteriol. 194 (2012) 6272-6281.

[26] T. Palmer, B.C. Berks, The twin-arginine translocation (Tat) protein export pathway, Nat. Rev. Microbiol. 10 (2012) 483-496.

[27] M. Muller, R.B. Klosgen, The Tat pathway in bacteria and chloroplasts (Review), Mol. Membr. Biol. 22 (2005) 113-121.

[28] D.A. Widdick, K. Dilks, G. Chandra, A. Bottrill, M. Naldrett, M. Pohlschroder, T. Palmer, The twin-arginine translocation pathway is a major route of protein export in Streptomyces coelicolor, Proc. Natl. Acad. Sci. USA 103 (2006) 1792717932.

[29] K. Schaerlaekens, M. Schierova, E. Lammertyn, N. Geukens, J. Anne, L. Van Mellaert, Twin-arginine translocation pathway in Streptomyces lividans, J. Bacteriol. 183 (2001) 6727-6732.

[30] M.G. Hicks, D. Guymer, G. Buchanan, D.A. Widdick, I. Caldelari, B.C. Berks, T. Palmer, Formation of functional Tat translocases from heterologous components, BMC Microbiol. 6 (2006).

[31] R.L. Jack, F. Sargent, B.C. Berks, G. Sawers, T. Palmer, Constitutive expression of Escherichia coli tat genes indicates an important role for the twin-arginine translocase during aerobic and anaerobic growth, J. Bacteriol. 183 (2001) 1801-1804.

[32] C. Dabney-Smith, H. Mori, K. Cline, Oligomers of Tha4 organize at the thylakoid Tat translocase during protein transport, J. Biol. Chem. 281 (2006) 5476-5483.

[33] M.C. Leake, N.P. Greene, R.M. Godun, T. Granjon, G. Buchanan, S. Chen, R.M. Berry, T. Palmer, B.C. Berks, Variable stoichiometry of the TatA component of the twin-arginine protein transport system observed by in vivo singlemolecule imaging, Proc. Natl. Acad. Sci. USA 105 (2008) 15376-15381.

[34] H. Mori, K. Cline, A twin arginine signal peptide and the $\mathrm{pH}$ gradient trigger reversible assembly of the thylakoid [Delta]pH/Tat translocase, J. Cell. Biol. 157 (2002) 205-210.

[35] J. Willemse, G.P. van Wezel, Imaging of Streptomyces coelicolor A3(2) with reduced autofluorescence reveals a novel stage of FtsZ localization, PLoS One 4 (2009) e4242.

[36] T. Kieser, M.J. Bibb, M.J. Buttner, K.F. Chater, D.A. Hopwood, Practical Streptomyces genetics, The John Innes Foundation, Norwich, United Kingdom, 2000.

[37] V. Jyothikumar, E.J. Tilley, R. Wali, P.R. Herron, Time-lapse microscopy of Streptomyces coelicolor growth and sporulation, Appl. Environ. Microbiol. 74 (2008) 6774-6781.

[39] J. Willemse, J.W. Borst, E. de Waal, T. Bisseling, G.P. van Wezel, Positive control of cell division: FtsZ is recruited by SsgB during sporulation of Streptomyces, Genes. Dev. 25 (2011) 89-99.

[40] D. Landgraf, B. Okumus, P. Chien, T.A. Baker, J. Paulsson, Segregation of molecules at cell division reveals native protein localization, Nat. Methods 9 (2012) 480-U498.

[41] B. Ruban-Osmialowska, D. Jakimowicz, A. Smulczyk-Krawczyszyn, K.F. Chater, J. Zakrzewska-Czerwinska, Replisome localization in vegetative and aerial hyphae of Streptomyces coelicolor, J. Bacteriol. 188 (2006) 7311-7316.

[42] M. Wolanski, R. Wali, E. Tilley, D. Jakimowicz, J. Zakrzewska-Czerwinska, P. Herron, Replisome trafficking in growing vegetative hyphae of Streptomyces coelicolor A3(2), J. Bacteriol. 193 (2011) 1273-1275. 